\title{
A Fuzzy Logic Algorithm for the Separation of Precipitating from Nonprecipitating Echoes Using Polarimetric Radar Observations
}

\author{
Jonathan J. Gourley,* Pierre Tabary, and Jacques Parent du Chatelet \\ Météo-France, Direction des Systèmes d'Observation, Trappes, France
}

(Manuscript received 11 January 2006, in final form 23 October 2006)

\begin{abstract}
A fuzzy logic algorithm has been developed for the purpose of segregating precipitating from nonprecipitating echoes using polarimetric radar observations at $\mathrm{C}$ band. Adequate polarimetric descriptions for each type of scatterer are required for the algorithm to be effective. An observations-based approach is presented in this study to derive membership functions and objectively weight them so that they apply directly to conditions experienced at the radar site and to the radar wavelength. Three case studies are examined and show that the algorithm successfully removes nonprecipitating echoes from rainfall accumulation maps.
\end{abstract}

\section{Introduction}

A major challenge in radar rainfall estimation is the detection and subsequent removal of nonprecipitating echoes such as ground clutter, sea clutter, clear air echoes, anomalous propagation of the beam, and chaff (i.e., thin, metallic-coated fibers released from military aircraft) from radar images. These targets have scattering characteristics that often resemble precipitating echoes. Errors in radar rainfall estimates have been shown to propagate nonlinearly in hydrologic forecasts of streamflow (Faures et al. 1995; Frank et al. 1999; Ogden et al. 2000; Droegemeier et al. 2000). The accuracy and timeliness of flash flood and river flood forecasts are limited by the accuracy of radar-derived precipitation.

Steiner and Smith (2002) provide an excellent summary of existing techniques to mitigate radar returns from ground clutter. They conclude that their algorithm yields improvement but fails in situations with strong, widespread clear air echoes and when anomalous propagation echoes are embedded in precipitation. Po-

* Additional affiliation: NOAA/National Severe Storms Laboratory, Norman, Oklahoma.

Corresponding author address: Jonathan J. Gourley, National Severe Storms Laboratory/National Weather Center, 120 David L. Boren Blvd., Rm. 4745, Norman, OK 73072.

E-mail: jj.gourley@noaa.gov larimetric radars transmit horizontally and vertically polarized waves, enabling them to measure the horizontal and vertical components of the backscatter amplitude. Additional information is provided by the magnitude and argument of the copolar cross-correlation coefficient between horizontal and vertical backscatter amplitudes. These polarimetric variables supply more information about the properties of meteorological and nonmeteorological scatterers compared to measurements from nonpolarimetric radars. To determine the benefits offered by polarimetry on improving the quality of rainfall accumulation products, an algorithm is developed that uses polarimetric variables alone. It is envisioned that future algorithms designed to improve data quality will integrate polarimetric observations into techniques that have already been developed using nonpolarimetric observations (e.g., Steiner and Smith 2002).

Algorithms designed to discriminate scatterers (e.g., ground clutter, precipitation) are based on thresholds, decision trees, fuzzy logic, neural networks, and combinations therein. Ryzhkov and Zrnic (1998) presented a threshold-based approach to rainfall estimation at $\mathrm{S}$ band when ground clutter is present. The identification of ground clutter was accomplished by establishing thresholds based on values of the cross-correlation coefficient $\left[\rho_{\mathrm{HV}}(0)\right]$ less than 0.7 . Values of the standard deviation of differential propagation phase measurements $\left(\Phi_{\mathrm{DP}}\right)$ within 17 consecutive range gates greater than $12^{\circ}$ were also used to detect ground clutter. Fuzzy 
logic algorithms readily accommodate radar data because polarimetric measurements, especially differential reflectivity $\left(Z_{\mathrm{DR}}\right)$ and $\Phi_{\mathrm{DP}}$, are inherently associated with noise. The magnitude of noise of polarimetric variables depends on many factors related to the radar hardware (e.g., antenna design, radome construction), its operating characteristics (e.g., pulse repetition frequency, dwell time), as well as the propagation and scattering characteristics of the targets (e.g., Doppler spectrum width). Fuzzy logic algorithms consider multiple radar observations; thus, the impact of a single noisy measurement is minimized. Algorithms have been used to identify ground clutter using Doppler radar data and to classify different hydrometeor types using polarimetric observations (Straka and Zrnic 1993; Holler et al. 1994; Straka 1996; Zrnic and Ryzhkov 1999; Vivekanandan et al. 1999; Liu and Chandrasekar 2000; Kessinger et al. 2001; Zrnic et al. 2001; May and Keenan 2005). Bringi and Chandrasekar (2001) present a detailed description of fuzzy logic algorithms, while Zrnic et al. (2001) highlight their differences.

The key to a successful fuzzy logic algorithm lies in the accuracy and applicability of functions used to describe the scatterers. Typically, qualitative knowledge acquired through simulations or observations concerning the range of values a scatterer possesses is formulated into a quantitative membership function. The membership functions in Zrnic et al. (2001) have trapezoidal shapes, while those of Liu and Chandrasekar (2000) are continuously differentiable beta functions. Once these functions have been derived for a given location, they may not be applicable to another radar that encounters different scatterers or may be operating at a different wavelength. A new, automated methodology of empirically deriving membership functions is developed in this study. While the membership functions derived in this study apply to C-band radars and the scatterers typically encountered near the Trappes radar (described below), the methodology of objectively defining membership functions is readily applicable at $\mathrm{X}, \mathrm{C}$, and $\mathrm{S}$ bands. It is unique in that it applies directly to the radar wavelength and characteristics specific to the radar site, such as beam propagation paths.

Membership functions provide a quantitative description of a given scatterer. The polarimetric properties of ground clutter, chaff, birds, and insects have been reported in the literature primarily at S-band frequency. Zrnic et al. (2006) found that, while mean values of $\rho_{\mathrm{HV}}(0)$ are generally lower in ground clutter than in precipitation, the distribution of $\rho_{\mathrm{HV}}(0)$ in ground clutter is broad and contains values that are commonly measured in precipitation. The use of $\rho_{\mathrm{HV}}(0)$ alone is inadequate to totally remove anomalous propagation and ground clutter echoes from reflectivity $\left(Z_{\mathrm{H}}\right)$ and $Z_{\mathrm{DR}}$ fields. The polarimetric properties of chaff have been described and compared to theoretical findings (Zrnic and Ryzhkov 2004). Chaff echoes are characteristic of values of $\rho_{\mathrm{HV}}(0)$ between 0.2 and $0.5, \Phi_{\mathrm{DP}}$ measurements of the receiver component of the system differential propagation phase, and values of $Z_{\mathrm{DR}}$ between 0 and $6 \mathrm{~dB}$ at $S$ band. Chaff is readily identified and thus separated from snow and rain echoes based on a simple threshold of $\rho_{\mathrm{HV}}(0)<0.6$. The polarimetric properties of birds and insects have been intensively studied (e.g., Wilson et al. 1994; Zrnic and Ryzhkov 1998; Zhang et al. 2005). Insects have ratios of horizontal-to-vertical cross sections of 3:1 and equivalent spherical diameters of $10 \mathrm{~mm}$. The differential reflectivity of insects has been observed to remain constant with viewing angle, whereas the differential reflectivity with birds varies between -2 and $4 \mathrm{~dB}$ at $\mathrm{S}$ band, depending on the size of the birds and the viewing angle. Mie scattering occurs with birds; thus, different values of $Z_{\mathrm{DR}}$ are expected at $\mathrm{X}$ and $\mathrm{C}$ bands.

The French national weather service, Météo-France, has been operating a C-band polarimetric radar in simultaneous transmission and reception mode continuously since the summer of 2004. The transmitted pulses have a width of $2 \mu \mathrm{s}$, a frequency of $5.64 \mathrm{GHz}$, a peak power of $250 \mathrm{~kW}$, and pulse repetition frequencies of 379, 321, and $305 \mathrm{~Hz}$. The 3-dB beamwidth of the 3.7m-diameter antenna is less than $1.1^{\circ}$. A detailed description of the radar system and the quality of the raw variables is provided in Gourley et al. (2006). The polarimetric variables measured by this radar are $Z_{\mathrm{H}}$, $Z_{\mathrm{DR}}, \rho_{\mathrm{HV}}(0)$, and $\Phi_{\mathrm{DP}}$. The radar is sited in flat terrain approximately $30 \mathrm{~km}$ to the southwest of Paris in Trappes, France. Weather services in Europe, Canada, and Japan are considering upgrading their radar networks with dual-polarimetric capabilities. The polarimetric properties of nonprecipitating echoes have been studied predominantly at S-band frequency. Thus, a major goal in this study is to derive membership functions (a key component to a fuzzy logic algorithm) based solely on polarimetric observations at $\mathrm{C}$ band.

The method of isolating scatterers and then empirically deriving their density functions is described in section 2. In section 3 a polarimetric fuzzy logic algorithm to identify nonprecipitating echoes and then remove them from rainfall rate and accumulation products is developed. Three case studies are examined in section 4 to evaluate its performance. A summary, conclusions, and suggestions for future work are provided in section 5 . 


\section{Characterization of precipitating and nonprecipitating echoes}

\section{a. Methodology}

The purpose of this section is to characterize the polarimetric signatures of precipitation and nonprecipitation echoes. These properties, which are expressed as objective functions, will then be used to ultimately identify nonprecipitation echoes and remove them from rainfall fields. The different types of nonprecipitation scatterers, also referred to as classes, are first identified. The choice of these classes depends on the nonprecipitation scatterers that are commonly encountered near the radar. In the case of the Trappes radar, the main contaminants to radar precipitation estimates are ground clutter from anomalous propagation of the beam, stationary ground clutter, and clear air echoes. Other sources, such as sea clutter, may need to be considered for coastal radars. Clutter from anomalous beam propagation is distinguished from stationary clutter by the farther ranges $(>10 \mathrm{~km})$ at which it is typically observed. Nonetheless, the scattering sources (e.g., trees, buildings, towers) are essentially the same; thus, they are treated jointly as a single class (referred to as ground clutter hereafter). Vertical refractivity gradients, insects, birds, and other biological targets such as bats have all been referred to as clear air echoes in the literature. A concise review of the origin of clear air echoes is provided in Wilson et al. (1994). While the term clear air echoes is used throughout this study, in this context it refers to insects. Chaff is occasionally observed near the Trappes radar. Its low frequency of occurrence has little impact on radar-based rainfall maps compared to echoes from ground clutter and clear air echoes, so a specific class for chaff is not required for this radar. However, it was noted that the scattering and propagation characteristics of chaff and solar radiation differ significantly from precipitation. Thus, in the event that these targets appear, they will be much more likely to be identified as either clear air echoes or ground clutter instead of precipitation.

First, radar measurements that exclusively represent ground clutter, clear air echoes, and precipitation are isolated. An example of radar reflectivity from ground clutter returns is shown in Fig. 1. Power lines, trees, buildings, and antennas are responsible for most of the echoes. Six hours of data (1400-2000 UTC 15 January 2005) at an elevation angle of $0.4^{\circ}$ (totaling 24 scans) were observed to be from ground clutter. These specific times of the day and year were chosen because radar images were completely devoid of clear air echoes and precipitation. The analysis was restricted to observations within $10 \mathrm{~km}$ of the radar to ensure that ground clutter alone was sampled.

Twenty-four hours of data collected at $1.5^{\circ}$ every 15 min on 25 March 2005 (totaling 96 scans) were used to characterize the polarimetric properties of clear air echoes. Processing such a large dataset was rather timeconsuming. Density functions describing the polarimetric properties of clear air echoes were monitored in order to determine how many scans were needed to adequately characterize the echoes. As it turns out, the density functions describing clear air echoes converged to their final values after only three scans were analyzed. The analysis was limited to ranges greater than $24 \mathrm{~km}$ but less than $50 \mathrm{~km}$. This placed the analysis domain beyond the ranges where ground clutter was noted and within the regions where clear air echoes were observed. Only three scans at $1.5^{\circ}$ in stratiform precipitation from the period 0700-0900 UTC 17 December 2004 and three scans of convective precipitation from 0700-0900 UTC 26 June 2005 were needed to estimate density functions for precipitation. Ranges in stratiform precipitation were required to be greater than $5 \mathrm{~km}$ in order to avoid ground clutter. Convective precipitation was observed within ranges of $50-200 \mathrm{~km}$.

In the next step, the spatial variability is calculated for $Z_{\mathrm{DR}}$ and $\Phi_{\mathrm{DP}}$. Here, the spatial variability is expressed as a root-mean-square difference or texture:

$$
\operatorname{Texture}\left(y_{a, b}\right)=\sqrt{\frac{\sum_{i=-(m-1) / 2}^{(m-1) / 2} \sum_{j=-(n-1) / 2}^{(n-1) / 2}\left(y_{a, b}-y_{a+i, b+j}\right)^{2}}{(m)(n)}},
$$

where $a$ and $b$ represent the azimuth and range of the gate. The texture of $Z_{\mathrm{DR}}$ and $\Phi_{\mathrm{DP}}(y)$ is computed in a box consisting of three pixels in azimuth direction $(m)$ and three in range direction $(n)$ centered on the gate.
Provided an azimuthal resolution of $0.5^{\circ}$ and a range resolution of $240 \mathrm{~m}$, the texture is representative of a $1.5^{\circ} \times 720 \mathrm{~m}$ area. This area increases with range; thus, the texture variable will have a range dependence. 


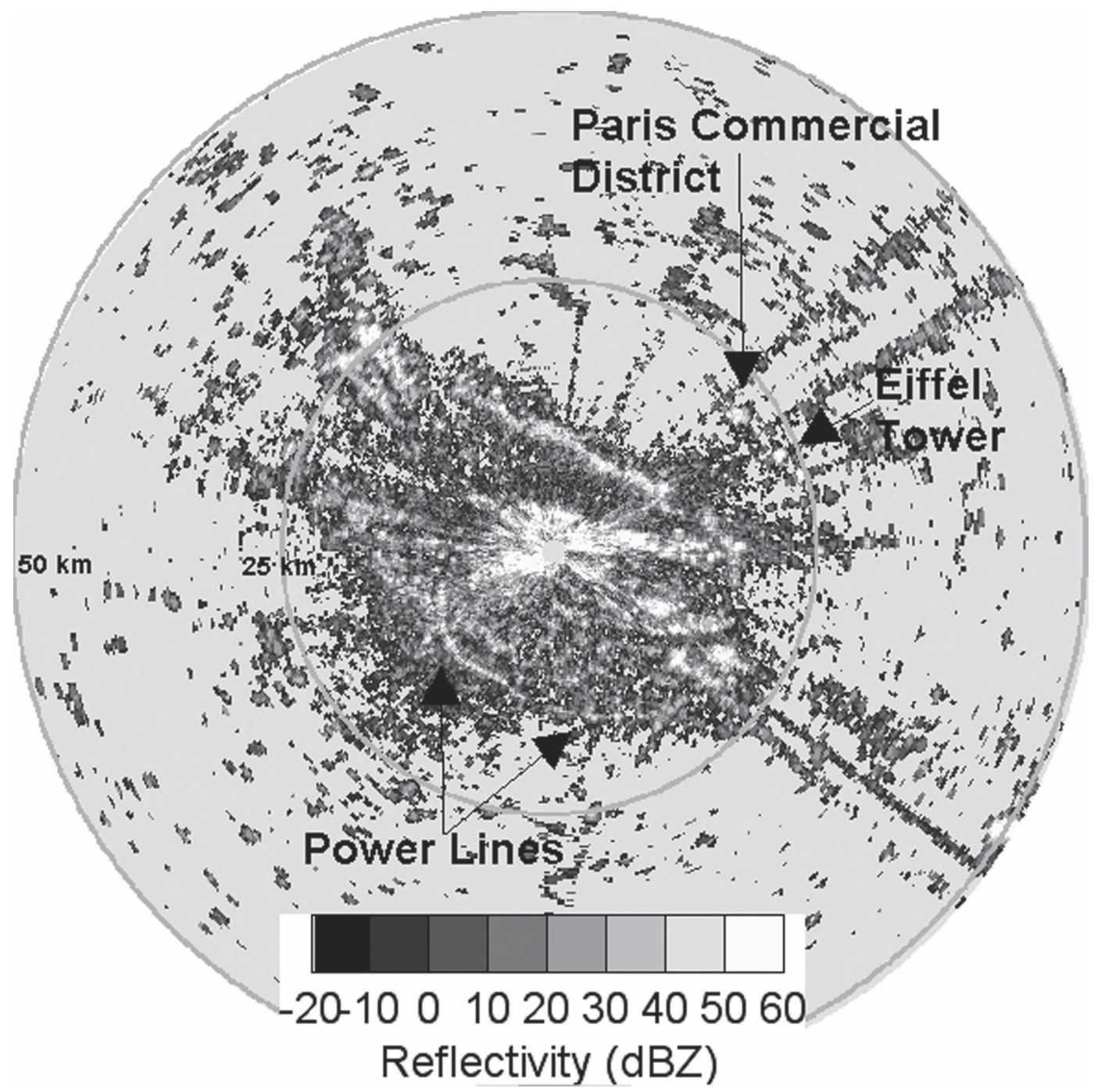

FIG. 1. Reflectivity factor $\left(Z_{\mathrm{H}}\right)$ in $\mathrm{dBZ}$ at an elevation angle of $0.4^{\circ}$ at $0800 \mathrm{UTC} 3 \mathrm{Jan} 2005$.

Figure 2 shows the average texture of $Z_{\mathrm{DR}}$ and $\Phi_{\mathrm{DP}}$ plotted as a function of range using 12 scans at $1.5^{\circ}$ from the period 0400-0500 UTC 4 July 2005. Up to approximately $45 \mathrm{~km}$, texture values decrease with range. Noisy values of $Z_{\mathrm{DR}}$ and $\Phi_{\mathrm{DP}}$ are more prevalent at close range, where ground clutter is encountered (see Fig. 1). Texture values are thus intrinsically higher close to radar. Beyond a range of approximately $45 \mathrm{~km}$, the texture values increase due to natural variability of $Z_{\mathrm{DR}}$ and $\Phi_{\mathrm{DP}}$ within the $1.5^{\circ} \times 720 \mathrm{~m}$ area, which also increases with range. The same dataset described above is used to empirically correct the range dependence of texture calculations for all cases analyzed hereafter. The correction procedure assumes that the textures of $Z_{\mathrm{DR}}$ and $\Phi_{\mathrm{DP}}$ increase linearly beyond a range of 45 $\mathrm{km}$. Lines are fit to the data shown in Fig. 2, and texture values measured beyond $45 \mathrm{~km}$ are subsequently re- duced to adjust for the range dependency. It is noted that the $45-\mathrm{km}$ range setting and the slope of the linear fit depend on the spatial structure of the precipitation as compared to the radar bin sizes. The range and degree of corrections that need to be applied to texture fields should be examined and optimized for precipitation events typically observed near the radar.

Next, density functions of $\rho_{\mathrm{HV}}(0)$, texture of $Z_{\mathrm{DR}}$, and texture of $\Phi_{\mathrm{DP}}$ are computed for each class (i.e., precipitation, ground clutter, and clear air echoes) using Gaussian kernel density estimation (Silverman 1986). The estimate of the data density is computed as follows:

$$
\hat{f}(x)=\frac{1}{\sigma \sqrt{2 \pi}} \sum_{i=1}^{n} e^{-\left[(1 / 2)\left(\frac{X_{i}-x}{\sigma}\right)^{2}\right]},
$$



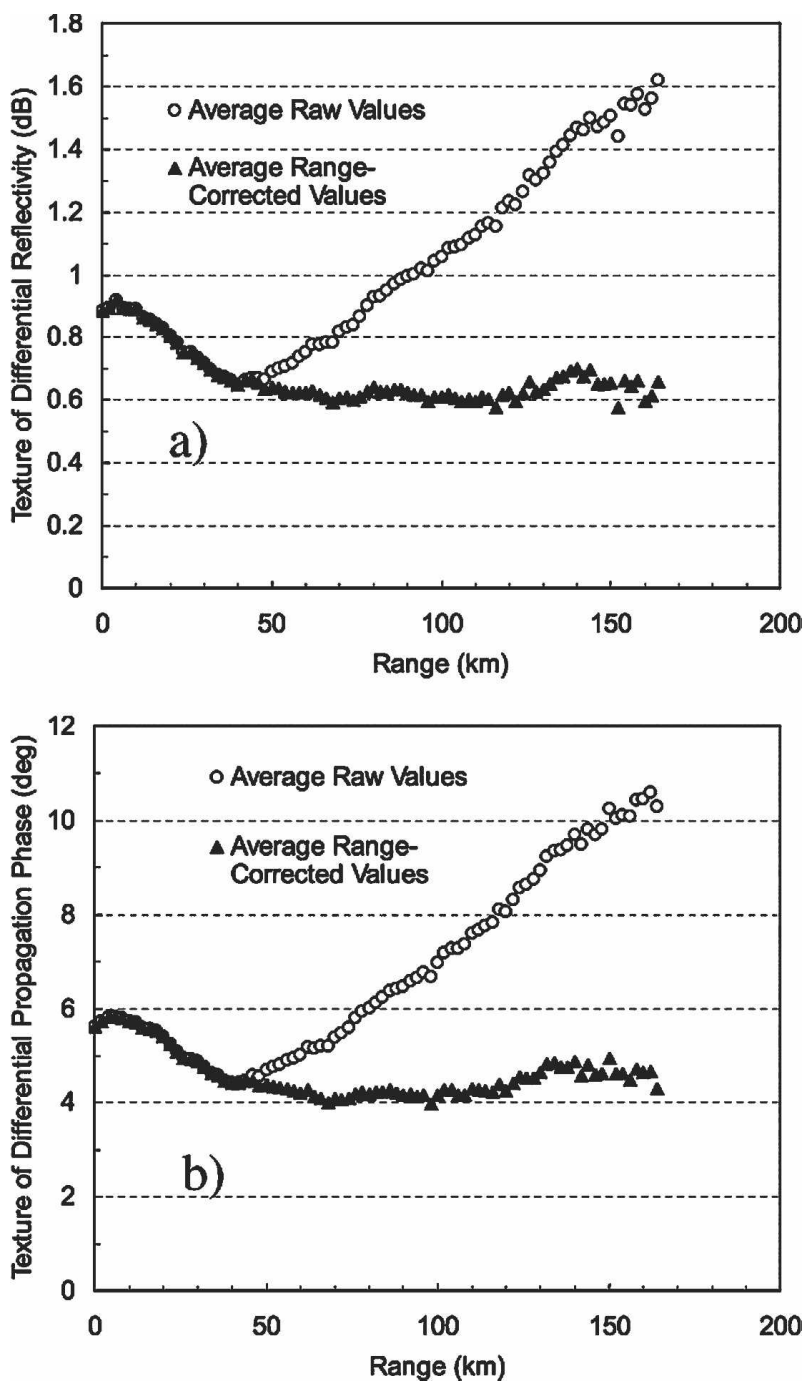

FIG. 2. Range dependence of (a) texture of differential reflectivity and (b) texture of differential propagation phase before and after empirical correction. Dataset is comprised of 12 scans at an elevation angle of $1.5^{\circ}$ from the period 0400-0500 UTC 4 Jul 2005.

where $\hat{f}(x)$ is the density function; $\sigma$ is the smoothing parameter or bandwidth; $n$ is the total number of data points; $X_{i}$ is the $i$ th observation of $\rho_{\mathrm{HV}}(0)$, texture of $Z_{\mathrm{DR}}$, or texture of $\Phi_{\mathrm{DP}}$; and $x$ is the independent variable $\left[\rho_{\mathrm{HV}}(0)\right.$, texture of $Z_{\mathrm{DR}}$, and texture of $\left.\Phi_{\mathrm{DP}}\right]$. The bandwidth controls the smoothness of the resulting function, where an estimate of the bandwidth is supplied here using Silverman's rule $\left(\sigma=1.06 \mathrm{SD} n^{-1 / 5}\right)$, where SD refers to the standard deviation computed from the actual distribution. The result of applying the Gaussian kernel is a function that is similar to a smoothed histogram. It has the advantage of being continuously differentiable, and the smoothing parameter, which is equivalent to the bin width in a histogram, is chosen objectively. All density functions are normalized so that the areas under each curve are equal to one.

\section{b. Results}

Figure 3 shows density functions of $\rho_{\mathrm{HV}}(0)$, texture of $Z_{\mathrm{DR}}$, and texture of $\Phi_{\mathrm{DP}}$ for precipitation, ground clutter, and clear air echoes. These three polarimetric variables are used in the fuzzy logic algorithm because their distributions in precipitation are distinct from their distributions in nonprecipitating echoes. A density function for nonprecipitating scatterers is computed by simply computing the average values for clear air echoes and ground clutter. The computed density function of $\rho_{\mathrm{HV}}(0)$ for precipitation has a mode of 0.97 , while the mode for clear air echoes is 0.42 (Fig. 3a). Moreover, the density function for $\rho_{\mathrm{HV}}(0)$ in precipitation has very little overlap with the density function representing clear air echoes. Therefore, the use of $\rho_{\mathrm{HV}}(0)$ in a fuzzy logic algorithm aids in the segregation between precipitation and clear air echoes. The density function of $\rho_{\mathrm{HV}}(0)$ for ground clutter has a mode of 0.92 and overlaps the density function of $\rho_{\mathrm{HV}}(0)$ for precipitation. Zrnic et al. (2006) also found significant overlap between precipitation and ground clutter histograms at $\mathrm{S}$ band. Density functions from additional variables are thus needed in order to effectively remove ground clutter from precipitating echoes.

The density function of the texture of $Z_{\mathrm{DR}}$ for precipitation has a mode of $0.4 \mathrm{~dB}$, while the modes of the clear air echoes and ground clutter distributions are 2.5 and $5.2 \mathrm{~dB}$, respectively (Fig. 3b). The ground clutter density function of the texture of $Z_{\mathrm{DR}}$ is well separated from precipitation, while there is more overlap between precipitation and clear air echo functions. The density function of the texture of $\Phi_{\mathrm{DP}}$ for precipitation has a mode of $4^{\circ}$, and values rarely exceed $20^{\circ}$ (Fig. 3c). The modes of the density functions of the texture of $\Phi_{\mathrm{DP}}$ for clear air echoes and ground clutter are $35^{\circ}$ and $125^{\circ}$ respectively. The precipitation density function is narrow and distinct from the nonprecipitating scatterers. The texture of $\Phi_{\mathrm{DP}}$ is a very useful parameter for identifying nonprecipitating echoes. All density functions shown in Fig. 3 are used collectively as membership functions in the fuzzy logic algorithm.

\section{Methodology of the polarimetric fuzzy logic algorithm}

The goal of this fuzzy logic algorithm is to incorporate polarimetric quantities [i.e., $\rho_{\mathrm{HV}}(0)$, texture of $Z_{\mathrm{DR}}$, and texture of $\left.\Phi_{\mathrm{DP}}\right]$ to determine whether the measurement was made in precipitation or was con- 

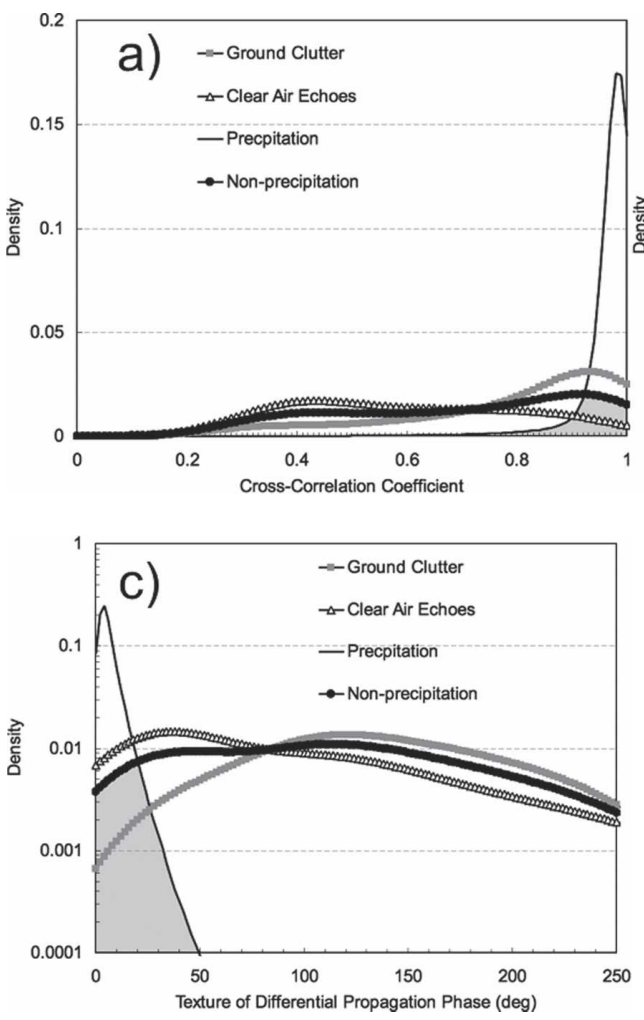

taminated by nonprecipitating echoes. It should be noted that no ground clutter rejection through spectral processing of Doppler velocities has been applied to raw $Z_{\mathrm{H}}$ data. The goal of this study is to examine the benefits offered by polarimetry to improving data quality. A schematic of the fuzzy logic algorithm is shown in Fig. 4. The classifier and ensuing terminology follows the hydrometeor particle identification algorithm used in Vivekanandan et al. (1999).

First, raw measurements of $Z_{\mathrm{H}}, Z_{\mathrm{DR}}, \rho_{\mathrm{HV}}(0), \Phi_{\mathrm{DP}}$, and radial velocity $\left(\mathbf{V}_{r}\right)$ are collected. Additional fields, such as the mean absolute difference in reflectivity between adjacent pulses at a given range gate (or sigma; Nicol et al. 2003), texture of $Z_{\mathrm{DR}}$, and texture of $\Phi_{\mathrm{DP}}$, are computed. At each pixel, the aggregation value $(Q)$ is calculated for each $i$ th class (i.e., clear air echoes, ground clutter, and precipitation) as follows:

$$
Q_{i}=\frac{\sum_{j=1}^{3} \hat{f}(x)_{j} \times W_{j}}{\sum_{j=1}^{3} W_{j}},
$$

where $\hat{f}(x)$ is the membership value for the $j$ th polarimetric quantity [i.e., $\rho_{\mathrm{HV}}(0)$, texture of $Z_{\mathrm{DR}}$, and texture of $\left.\Phi_{\mathrm{DP}}\right]$. Equation (3) enables the polarimetric

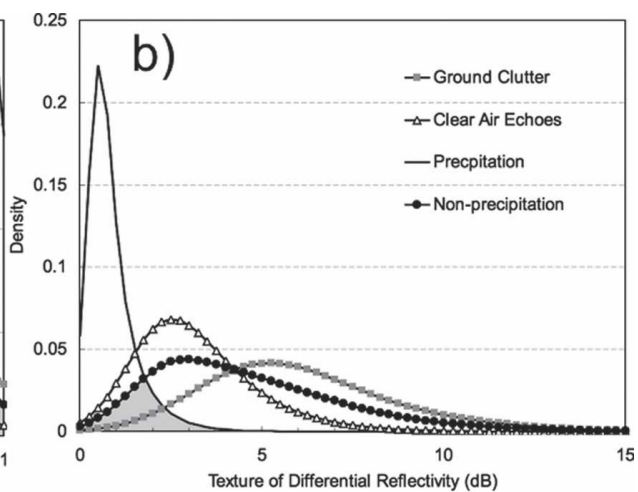

FIG. 3. Empirically estimated density functions $[\hat{f}(x)]$ of (a) cross-correlation coefficient $\left[\rho_{\mathrm{HV}}(0)\right]$, (b) texture of differential reflectivity $\left(Z_{\mathrm{DR}} ; \mathrm{dB}\right)$, and (c) texture of differential propagation phase $\left(\Phi_{\mathrm{DP}} ;{ }^{\circ}\right)$ for the classes indicated on the plots. Density functions were derived using observations from a $\mathrm{C}$-band polarimetric radar and serve directly as membership functions in a fuzzy logic algorithm. The shaded regions (in gray) showing the area overlap between nonprecipitation and precipitation classes control the weight applied to each variable.

quantities to be weighted by $W$. The weights are determined objectively by first computing the area overlap between precipitation and nonprecipitation scatterers, as in Cho et al. (2006). A membership function for nonprecipitation scatterers was calculated by averaging the membership functions representing clear air echoes and ground clutter. If it were desirable to distinguish between the different types of nonweather scatterers (i.e., clear air echoes versus ground clutter), then an array of weights would be needed for each variable and each class comparison. This study is merely aimed at segregating precipitation from nonprecipitating echoes; thus, a single weight is used for each polarimetric variable. The overlap areas between precipitation and nonprecipitation (shaded in gray in Fig. 3) are equal to $0.243,0.203$, and 0.081 for $\rho_{\mathrm{HV}}(0)$, texture of $Z_{\mathrm{DR}}$, and texture of $\Phi_{\mathrm{DP}}$, respectively. These areas are used to compute the weights as follows:

$$
W_{j}=\frac{1}{A_{j}} \sum_{j=1}^{3} \frac{1}{A_{j}} .
$$

Thus, the texture of $\Phi_{\mathrm{DP}}$ has 3 times more weight than $\rho_{\mathrm{HV}}(0)$ and more than double the weight of the texture of $Z_{\mathrm{DR}}$.

The membership values are simply looked up from the membership functions provided in Fig. 3. For in- 

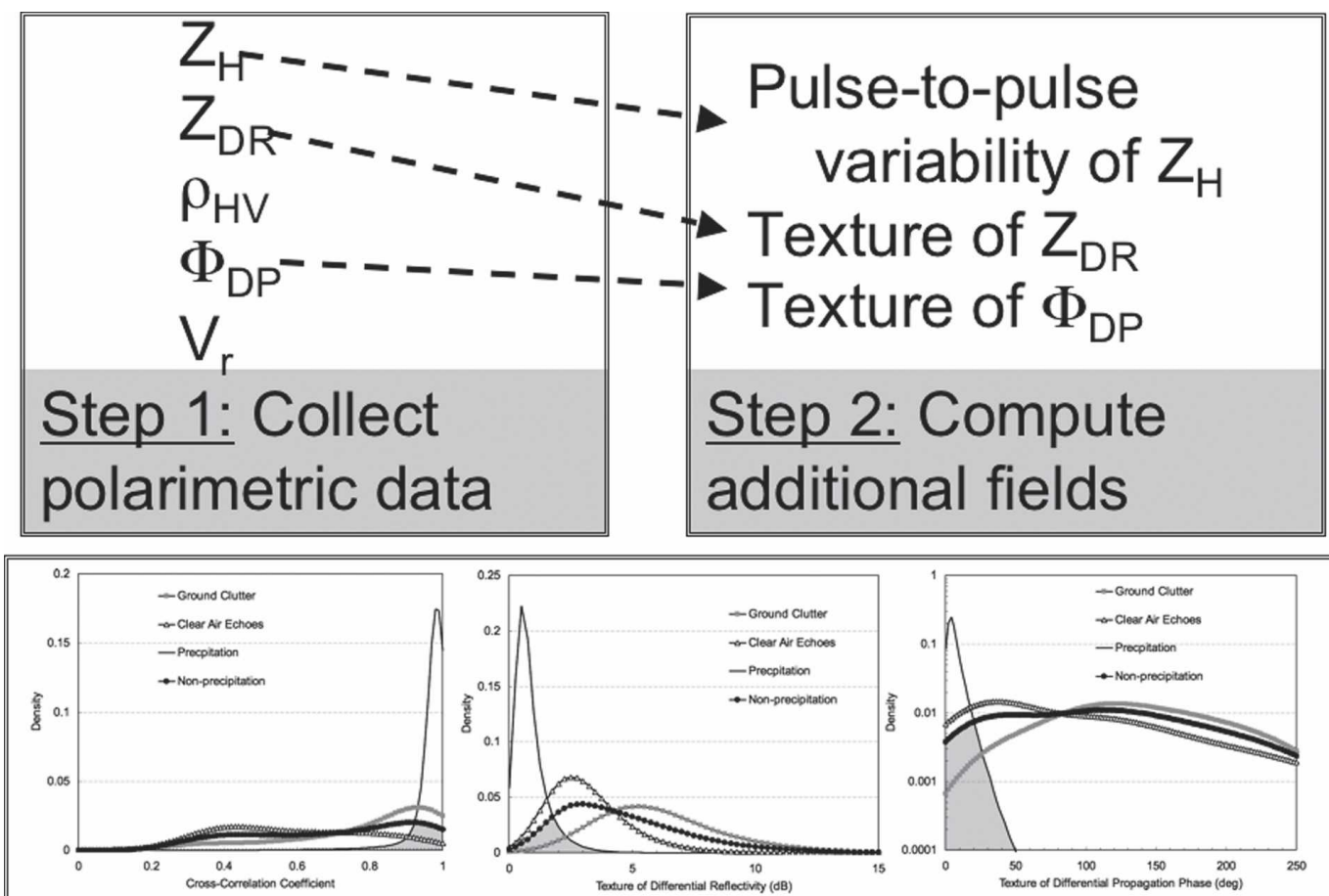

\section{Step 3: Lookup membership value}

Step 4: Compute class

Class $\sim \operatorname{MAX}\left(Q_{c a}, Q_{g c}, Q_{p p t}\right)$

Step 5: Reject class assignments using Table 1

\section{Step 6: Despeckle class fields}

FIG. 4. Schematic of the polarimetric fuzzy logic algorithm to separate precipitating from nonprecipitating echoes.

stance, if the observed $\rho_{\mathrm{HV}}(0)$ is 0.97 , then the membership value of the pixel $\left[\hat{f}(x)_{1}\right]$ being precipitation is $0.149,0.030$ for ground clutter, and 0.007 for clear air echoes. The weighted aggregation value $(Q)$ is then calculated for each $i$ th class given membership values corresponding to $\rho_{\mathrm{HV}}(0)$, the texture of $Z_{\mathrm{DR}}$, and texture of $\Phi_{\mathrm{DP}}$ [i.e., $\hat{f}(x)_{1}, \hat{f}(x)_{2}$, and $\hat{f}(x)_{3}$ ]. The class is then determined by comparing the weighted aggregation values. The class is assigned to the pixel based on the maximum aggregation value. While the fuzzy logic algorithm has the capability to segregate between clear air echoes and ground clutter by applying appropriate weights, the primary intention of the algorithm is to separate precipitation from nonprecipitation echoes. For this reason, results in section 4 are presented by combining clear air echoes and ground clutter into a single, nonprecipitation echoes class.
The approach of taking the maximum aggregation value differs from the fuzzy logic classifiers used in Kessinger et al. (2001) and Cho et al. (2006). Both studies use empirical curves obtained for the condition of a pixel belonging to a class given radar measurements. A membership value for a given class is then determined by normalizing $\hat{f}(x)_{j}$ by the sum of $\hat{f}(x)_{j}$ values obtained for all three classes. This normalization process is consistent with the statistical model discussed in Bringi and Chandrasekar (2001). It is noted, however, that the aforementioned classifiers establish thresholds for a pixel belonging to a class as opposed to taking the maximum aggregation value, as is done in this study. When the maximum aggregation value is chosen, as proposed herein, normalizing membership values by a constant has no effect on the final class determination.

A brief discussion regarding the use of aggregation 
TABLE 1. Empirical thresholds to suppress erroneous class assignments for precipitation, ground clutter, and clear air echoes.

\begin{tabular}{lll}
\hline \hline \multicolumn{1}{c}{ Variables } & Thresholds & $\begin{array}{c}\text { Suppressed class } \\
\text { assignment }\end{array}$ \\
\hline$\rho_{\mathrm{HV}}(0)$ & $<0.7$ & Precipitation \\
$\Phi_{\mathrm{DP}}$ & $<-40^{\circ}$ & Precipitation \\
$Z_{\mathrm{H}}$ & $<5 \mathrm{dBZ}$ & Precipitation \\
Texture of $\Phi_{\mathrm{DP}}$ & $>100^{\circ}$ & Precipitation \\
Pulse-to-pulse variability of $Z_{H}$ & $>5 \mathrm{~dB}$ & Ground clutter \\
Radial velocity & $>5 \mathrm{~m} \mathrm{~s}^{-1}$ & Ground clutter \\
$Z_{\mathrm{H}}$ & $>30 \mathrm{dBZ}$ & Clear air echoes \\
\hline
\end{tabular}

values, as in Zrnic et al. (2001), versus multiplicative values, as in Liu and Chandrasekar (2000), is warranted. In a multiplicative fuzzy logic algorithm, a membership value of zero computed from a single membership function for a given class will prohibit that class from being assigned, even if the measured variable (e.g., texture of $Z_{\mathrm{DR}}$ ) was in error. By weighting and summing the values, a class can still be assigned even if an individual membership value is zero. The additive approach thus considers inherent errors associated with radar measurements (e.g., noise, partial beam filling, reduction of reflectivity with height, miscalibration, radome wetting effects, etc.). The aggregated values, however, are more similar for different classes compared to values obtained by multiplication. This means that there is a greater chance that classes will be assigned inappropriately using aggregated values. Thresholds are thus applied based on the variables listed in Table 1 where it is certain a given class must not be assigned. The application of these thresholds overrules the original classification made by the fuzzy logic algorithm. In effect, the application of thresholds is equivalent to assigning a zero membership value in the Liu and Chandrasekar (2000) scheme. The use of thresholds represents a compromise between fuzzy logic algorithms that maximize the sum versus the product. These thresholds were tuned to the Trappes Cband radar and may need to be optimized for other radars.

The classification fields are produced on a 1066 pixel $\times 720$ pixel grid corresponding to a resolution of $240 \mathrm{~m} \times 0.5^{\circ}$. The fields are resampled on a 512 pixel $\times$ 512 pixel Cartesian grid having a resolution of $1 \mathrm{~km} \times$ $1 \mathrm{~km}$. A despeckling algorithm is then applied to the classification field based on the following rules. A $3 \times$ 3 region surrounding each pixel is examined. If the central pixel is determined to be precipitation and less than three of the neighboring pixels are identified as precipitation, then the central pixel is assumed to be isolated and is reassigned to the clear air echo class. If the central pixel is determined to be clear air echo and more than six of the surrounding pixels are precipitation pixels, then the clear air echo designation is reassigned to the precipitation class. This despeckling step produces spatial continuity in the resulting classification fields.

Figure 5 shows radar reflectivity, $\rho_{\mathrm{HV}}(0)$, texture of $Z_{\mathrm{DR}}$, texture of $\Phi_{\mathrm{DP}}$, and the resulting classification for a single scan at $1.5^{\circ}$ at 0745 UTC 23 June 2005. Analysis of the reflectivity image shows ground clutter with $Z_{\mathrm{H}}$ values as high as $60 \mathrm{~dB} Z$ within $10 \mathrm{~km}$ of the radar and clear air echoes with $Z_{\mathrm{H}}<0 \mathrm{~dB} Z$ within $60 \mathrm{~km}$. Reflectivity values exceeding $40 \mathrm{dBZ}$ from anomalous propagation were observed to the northwest of the radar. Precipitation with $Z_{\mathrm{H}}$ between 5 and $35 \mathrm{~dB} Z$ was organized in a band to the southwest and southeast of the radar at ranges between 80 and $150 \mathrm{~km}$. Values of $\rho_{\mathrm{HV}}(0)$ were at least 0.97 in the precipitating echoes, while they were approximately $0.3-1.0$ and $0.4-1.0$ in ground clutter/anomalous propagation and clear air echo echoes, respectively. The wide range of $\rho_{\mathrm{HV}}(0)$ values observed in nonprecipitating echoes requires additional information provided by the texture of $Z_{\mathrm{DR}}$ and texture of $\Phi_{\mathrm{DP}}$ fields to adequately separate them from precipitating echoes. Values of the texture of $Z_{\mathrm{DR}}$ in the light rain region were less than $0.5 \mathrm{~dB}$, while they were greater than $1.0 \mathrm{~dB}$ in ground clutter. Clear-air echoes, however, do not exhibit high spatial variability in $Z_{\mathrm{DR}}$. Values of the texture of $Z_{\mathrm{DR}}$ in clear air echoes are similar to those observed in precipitation. The texture of $\Phi_{\mathrm{DP}}$ images shows that values in precipitation are less than $5^{\circ}$, while they range from $10^{\circ}$ to $100^{\circ}$ in nonprecipitating echoes. The classification field was then computed using the proposed fuzzy logic algorithm. The results indicate that the nonprecipitating echoes were properly identified. It is noted that clear air echoes were identified in a small region enveloping the precipitation. The close proximity of these echoes to the precipitation region suggests that their classification as being clear air echoes is not entirely accurate. Polarimetric quantities measured at low signal-to-noise ratio are more prone to errors. In addition, the computed texture fields are subject to edge effects in these regions. Nonetheless, reflectivity values are less than 5 $\mathrm{dB} Z$, which is too small to contribute to rainfall-rate estimates. From the perspective of identifying and removing nonprecipitating echoes, the fuzzy logic algorithm was successful for this particular scan.

\section{Results}

The previous section demonstrates how the polarimetric fuzzy logic algorithm performed for a single scan. The efficacy of the algorithm is evaluated more thoroughly in this section by examining three different 

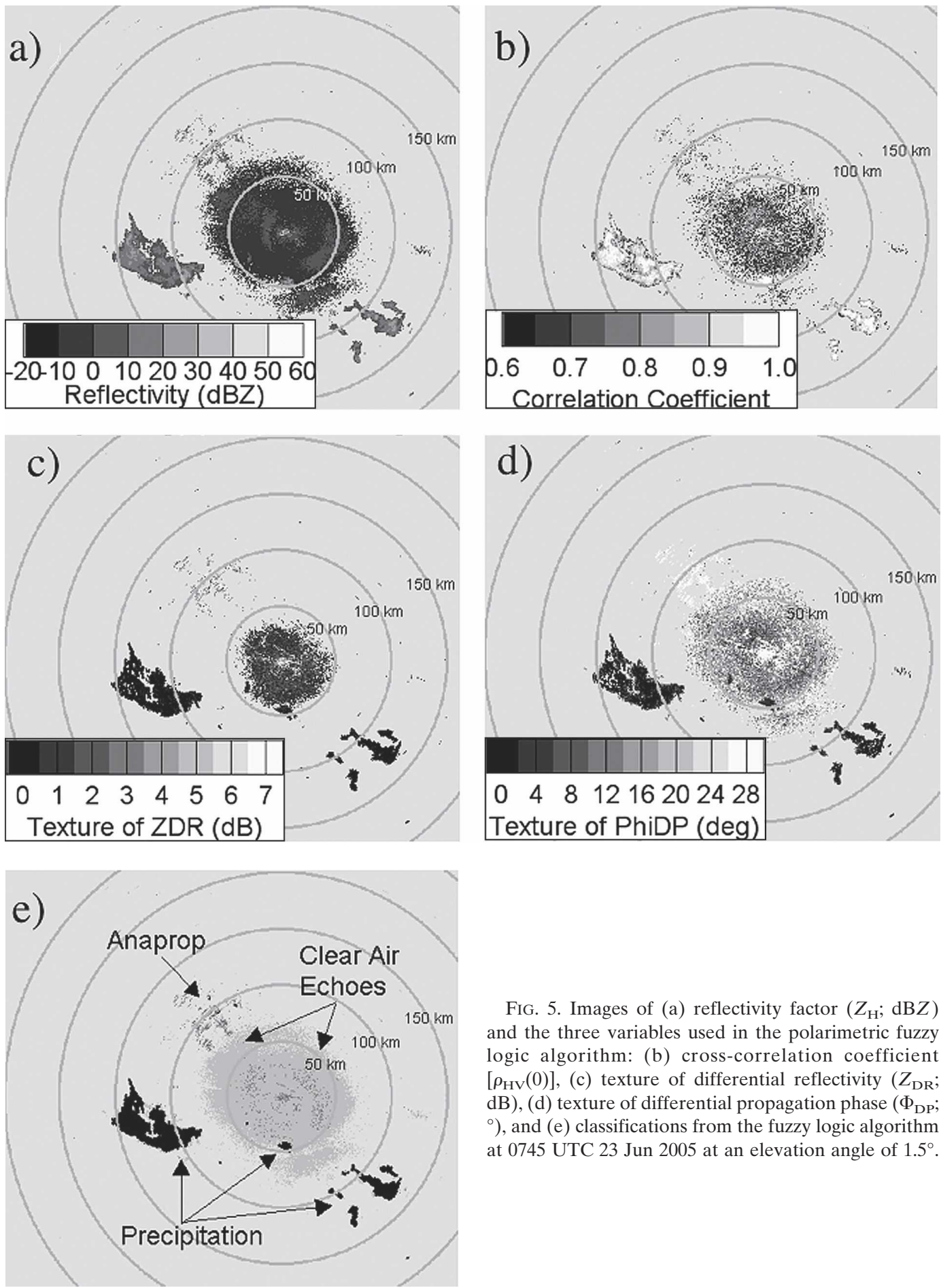

FIG. 5. Images of (a) reflectivity factor $\left(Z_{\mathrm{H}} ; \mathrm{dBZ}\right)$ and the three variables used in the polarimetric fuzzy logic algorithm: (b) cross-correlation coefficient $\left[\rho_{\mathrm{HV}}(0)\right],(\mathrm{c})$ texture of differential reflectivity $\left(Z_{\mathrm{DR}}\right.$; $\mathrm{dB}),(\mathrm{d})$ texture of differential propagation phase $\left(\Phi_{\mathrm{DP}}\right.$; ${ }^{\circ}$ ), and (e) classifications from the fuzzy logic algorithm at 0745 UTC 23 Jun 2005 at an elevation angle of $1.5^{\circ}$.

cases that span 1-24 h. Reflectivity values in the algorithm-identified precipitating regions are converted to rainfall-rate estimates using the Marshall-Palmer $Z-R$ relationship (i.e., $Z=200 R^{1.6}$ ) and accumulated over the event duration (Marshall and Palmer 1948). To assess the potential impact of nonprecipitating echoes on rainfall accumulations, reflectivity data from algorithmidentified nonprecipitating echoes are converted to rain rates and accumulated in the same manner as precipitating echoes. The sum of the precipitation and nonprecipitation accumulation fields shows the result if no data quality checks were performed on reflectivity data. 
The accumulation maps from precipitating and nonprecipitating echoes are then compared for a case that had no precipitation but significant ground clutter and anomalous propagation, a predominately widespread rainfall case with a small region of ground clutter, and a case with intense convection, clear air echoes, and ground clutter. It is recognized that this evaluation methodology does not have the benefit of a ground truth dataset. However, the evaluation is performed over several hours for cases that were carefully studied in order to understand the various sources of the echoes.

\section{a. A nonprecipitation event with ground clutter and anomalous propagation}

Classification results were produced every $15 \mathrm{~min}$ from 1400 to 2000 UTC 15 January 2005 using data collected at an elevation angle of $0.4^{\circ}$. Visible satellite imagery was inspected to verify that indeed there were no clouds in the vicinity of the radar. Accumulations from algorithm-identified precipitating echoes and potential accumulations from nonprecipitating echoes are compared. The impact of reflectivity from algorithmidentified ground clutter and anomalous propagation on rainfall estimates is shown in Fig. 6a. Rainfall estimates exceeding $200 \mathrm{~mm}$ would have accumulated within $10 \mathrm{~km}$ of the radar if echoes from ground clutter were not adequately removed. Echoes from anomalous propagation would have resulted in rain accumulations as high as $5 \mathrm{~mm}$ in an arc to the west of the radar at a range of approximately $100 \mathrm{~km}$. A similar region of contamination exists to the north of the radar between ranges of $170-200 \mathrm{~km}$. The polarimetric fuzzy logic algorithm could be judged as perfectly skillful only if there were no accumulations from precipitation echoes. Accumulations from algorithm-identified precipitating echoes are very close to zero except for isolated pixels and a small region to the north of the radar (Fig. 6b). A closer inspection of the accumulations in these regions revealed that the accumulations are a fraction of $1 \mathrm{~mm}$ and are negligible.

\section{b. A widespread rainfall case with a small region of ground clutter}

The polarimetric fuzzy logic algorithm classified precipitating and nonprecipitating echoes for 12 scans of data at an elevation angle of $1.5^{\circ}$ from the period 0400 0500 UTC 4 July 2005. Movie loops of the polarimetric variables were analyzed to aid in determining the sources of the echoes. This case was dominated by widespread rainfall, but a small region of ground clutter was noted near the radar. Echoes from ground clutter were identified from the movie loops because they remained stationary. Nonprecipitating echoes were then segregated from precipitating echoes using the automated fuzzy logic algorithm. Figure 6c shows rainfall accumulations that would have resulted from nonprecipitating echoes. Isolated pixels from ground clutter within $10 \mathrm{~km}$ of the radar would have contributed as much as $100 \mathrm{~mm}$ to an hourly rainfall accumulation map.

Nonprecipitating echoes were incorrectly identified on the outer fringes of raining regions between 200 and $250 \mathrm{~km}$ in range. Beam heights exceeded $5 \mathrm{~km}$ at these long ranges; thus, the radar was observing reflectivity values $<10 \mathrm{dBZ}$ associated with ice. As shown in Table 1 , precipitation classifications are not permitted with echoes that have reflectivity $<5 \mathrm{~dB} Z$. In effect, the polarimetric fuzzy logic algorithm and perhaps the polarimetric quantities themselves were useful up to a range of approximately $200 \mathrm{~km}$ with this case. The range at which the algorithm is effective improves with lower radar elevation angles and with deep, convective storms that are more visible to radar at far range.

Figure $6 \mathrm{~d}$ shows accumulations from reflectivity observed in algorithm-identified precipitating echoes. The pattern of accumulations reveals no noticeable artifacts that would have resulted from nonprecipitating echoes that were not adequately removed. Some isolated pixels within $5 \mathrm{~km}$ of the radar, however, have zero accumulations. These pixels were identified as ground clutter and thus created small holes in the rainfall accumulation field. The signals at these pixels were mixed between precipitation and ground clutter. Spectral processing methods will be necessary to separate the precipitation versus nonprecipitation characteristics of the spectrum. Otherwise, these holes can be simply mitigated by spatially interpolating data from nearby pixels that were classified as precipitation.

\section{c. A case with intense convection, clear air echoes, and ground clutter}

The fuzzy logic algorithm identified nonprecipitating and precipitating echoes for 96 scans of data collected at an elevation angle of $1.5^{\circ}$ from the period 0000-2345 UTC 23 June 2005. Figure 6e shows accumulations from ground clutter, clear air, and solar radiation. Reflectivity from ground clutter within $5 \mathrm{~km}$ of the radar results in rainfall accumulations exceeding $200 \mathrm{~mm}$ at several pixels. Reflectivity from clear air echoes results in accumulations generally less than $1 \mathrm{~mm}$ extending out to a range of $70 \mathrm{~km}$. The impact of clear air echoes 
Non-precipitating echoes
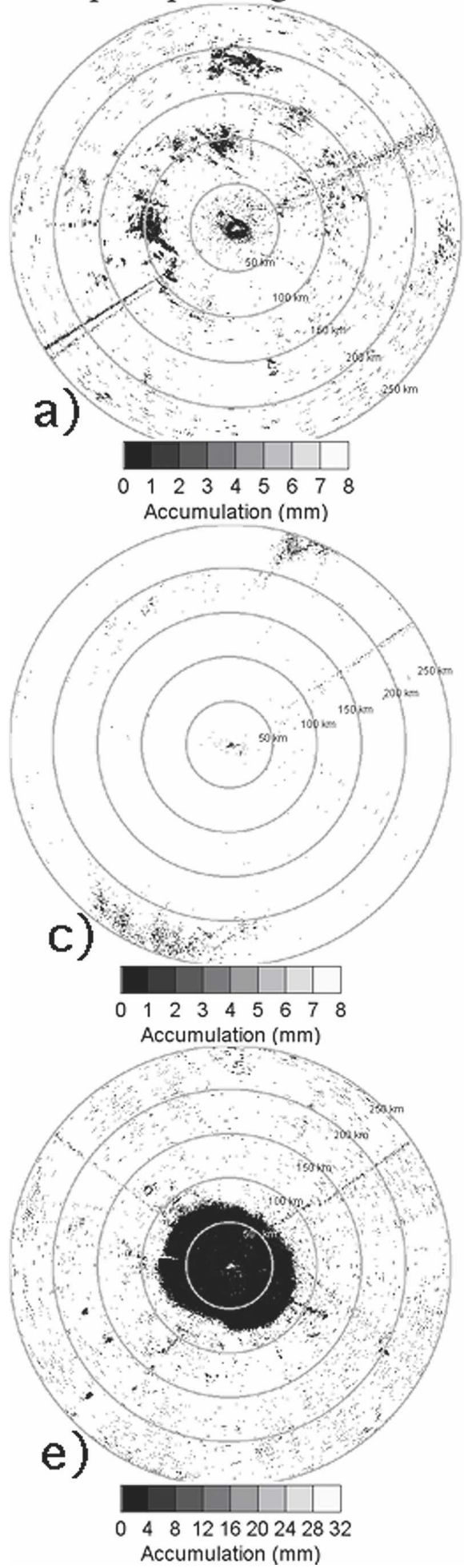

Precipitating echoes
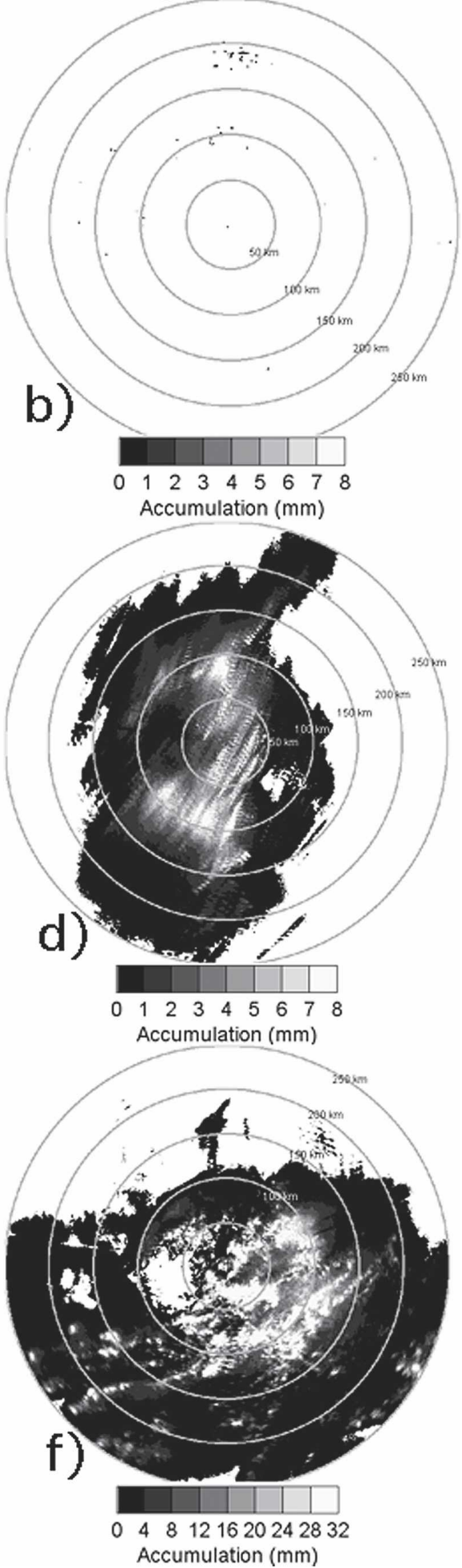

FIG. 6. Radar estimates of rainfall (in $\mathrm{mm}$ ) from algorithm-identified (a) nonprecipitating echoes and (b) precipitating echoes from the period 1400-2000 UTC 15 Jan 2005 using data at an elevation angle of $0.4^{\circ}$, (c) nonprecipitating echoes and (d) precipitating echoes from 0400-0500 UTC Jul 2005 using data at an elevation angle of $1.5^{\circ}$, and (e) nonprecipitating echoes and (f) precipitating echoes from 0000-2345 UTC 23 Jun 2005 using data at an elevation angle of $1.5^{\circ}$. Rainfall estimates were computed using $Z=200 R^{1.6}$. 
on radar rainfall estimates is not as significant as with ground clutter, but these echoes cover a large region and will bias long-term (e.g., daily or monthly) rainfall accumulations. Even though a specific class was not designated for solar radiation, artifacts from solar radiation are noted along radials extending to the northwest and northeast of the radar. The polarimetric characteristics of solar radiation resembled clear air echoes more so than precipitation. Lastly, accumulations from nonprecipitating echoes appear to have a noisy appearance. Some of these echoes are due to aircraft, but a majority is likely due to system noise. Accumulations from precipitating echoes, on the other hand, depict no noticeable artifacts near the radar resulting from clear air echoes, ground clutter, or solar radiation (Fig. 6f). Moreover, the precipitation accumulation map does not appear noisy in the northern part of the radar umbrella where there was no rain. This shows that the despeckling algorithm effectively removes isolated pixels that were mistakenly identified as precipitation from noisy polarimetric observations.

\section{Summary and conclusions}

A fuzzy logic algorithm supplied with polarimetric radar observations has been developed to identify and remove nonprecipitating echoes from rainfall accumulation maps. The definition of the membership functions, which up to now have been based on simulations or manual observation, has a strong influence on the overall performance of a fuzzy logic algorithm. A methodology was devised in this paper to compute density functions of polarimetric variables and their textures for scatterers that are known to be from precipitation and nonprecipitating sources (e.g., anomalous propagation, ground clutter, and clear air echoes). The empirically derived density functions using Gaussian kernel density estimation serve directly as membership functions in a fuzzy logic algorithm. The weights applied to each membership function are chosen objectively, as in Cho et al. (2006), using the area overlap between precipitation and nonprecipitation classes. This automatic methodology to derive membership functions in fuzzy logic algorithms offers the following advantages:

- It is based entirely on observations, is objective, and thus may be readily applied to any type of scanning radar.

- Derived membership functions apply directly to the radar wavelength and to propagation characteristics of the beam specific to the radar site.

- It works automatically without the need to adjust parameter settings.
To evaluate the performance of the fuzzy logic algorithm, three cases were studied, from which the following conclusions have been drawn:

- The distribution of the spatial variability of $\Phi_{\mathrm{DP}}$ (as defined by a texture parameter) for precipitation echoes is well separated from the density function representing nonprecipitation echoes. This variable is very useful in segregating precipitating from nonprecipitating echoes. The texture of $Z_{\mathrm{DR}}$ and $\rho_{\mathrm{HV}}(0)$ also aid in discriminating common contaminants to radar-based rainfall products.

- The use of objectively chosen weights for the polarimetric variables and their classes significantly improved the skill of the algorithm.

- Outer edges of precipitating echoes were occasionally classified as clear air echoes. This edge effect was more problematic at ranges greater than $200 \mathrm{~km}$ from radar. The effect on rainfall accumulations, however, is small because $Z_{\mathrm{H}}$ is generally less than $5 \mathrm{~dB} Z$.

- The algorithm successfully removes ground clutter, anomalous propagation, solar radiation, noise, and clear air echoes from radar rainfall estimates.

- Handling of mixed signals, such as ground clutter and anomalous propagation echoes embedded in precipitation, may require spectral approaches using polarimetric measurements.

Areas inviting future research are evaluating the developed fuzzy logic algorithm for additional cases including anomalous propagation echoes embedded in precipitation, deriving membership functions, and applying the algorithm on a polarimetric radar sited in a different regime and operating at S- and X-band frequencies, integrating the capabilities to identify ground clutter using previously developed techniques for nonpolarimetric radars, and extending the methodology of objectively deriving membership functions to hydrometeor classification algorithms (e.g., hail, wet snow, pristine ice, rain, etc.).

Acknowledgments. This work was done in the frame of the Programme ARAMIS Nouvelles Technologie en Hydrometeorologie Extension et Renouvellement (PANTHERE) Project supported by Météo-France, the French Ministere de L'Ecologie et du Developpement Durable, the European Regional Development Fund (ERDF) of the European Union, and CEMAGREF. The authors would like to acknowledge the advice provided by the PANTHERE scientific review committee. Specifically, Drs. Anthony Illingworth and Martin Hagen provided useful comments at several different stages throughout the development of this algorithm. The comments and suggestions provided by Dr. Katja 
Friedrich of MeteoSwiss and two anonymous reviewers improved the figures and quality of this manuscript. Their assistance is greatly appreciated.

\section{REFERENCES}

Bringi, V. N., and V. Chandrasekar, 2001: Polarimetric Doppler Weather Radar: Principles and Applications. Cambridge University Press, $636 \mathrm{pp}$.

Cho, Y.-H., G. W. Lee, K.-E. Kim, and I. Zawadzki, 2006: Identification and removal of ground echoes and anomalous propagation using the characteristics of radar echoes. J. Atmos. Oceanic Technol., 23, 1206-1222.

Droegemeier, K. K., and Coauthors, 2000: Hydrological aspects of weather prediction and flood warnings: Report on the Ninth Prospectus Development Team of the U.S. Weather Research Program. Bull. Amer. Meteor. Soc., 81, 2665-2680.

Faures, J. M., D. C. Goodrich, D. A. Woolhiser, and S. Sorooshian, 1995: Impact of small-scale spatial rainfall variability on runoff simulation. J. Hydrol., 173, 309-326.

Frank, E., M. Borga, and E. N. Anagnostou, 1999: Hydrological modeling of mountainous basins using radar rainfall data. Preprints, 29th Int. Conf. on Radar Meteorology, Montreal, QC, Canada, Amer. Meteor. Soc., 717-720.

Gourley, J. J., P. Tabary, and J. Parent du Chatelet, 2006: Data quality of the Meteo-France C-band polarimetric radar. $J$. Atmos. Oceanic Technol., 23, 1340-1356.

Holler, J., V. N. Bringi, J. Hubbert, M. Hagen, and P. F. Meischner, 1994: Life cycle and precipitation formation in a hybrid-type hailstorm revealed by polarimetric and Doppler radar measurements. J. Atmos. Sci., 51, 2500-2522.

Kessinger, C., S. Ellis, and J. VanAndel, 2001: NEXRAD data quality: The AP clutter mitigation scheme. Preprints, 30th Int. Conf. on Radar Meteorology, Munich, Germany, Amer. Meteor. Soc., 707-709.

Liu, H., and V. Chandrasekar, 2000: Classification of hydrometeors based on polarimetric radar measurements: Development of fuzzy logic and neuro-fuzzy systems, and in situ verification. J. Atmos. Oceanic Technol., 17, 140-164.

Marshall, J. S., and W. M. Palmer, 1948: The distribution of raindrops with size. J. Atmos. Sci., 5, 165-166.

May, P. T., and T. D. Keenan, 2005: Evaluation of microphysical retrievals from polarimetric radar with wind profiler data. $J$. Appl. Meteor., 44, 827-838.

Nicol, J. C., P. Tabary, J. Sugier, J. Parent du Chatelet, and G. Delrieu, 2003: Non-weather echo identification for conventional operational radar. Preprints, 31st Int. Conf. on Radar Meteorology, Seattle, WA, Amer. Meteor. Soc., 542-545.
Ogden, F. L., H. O. Sharif, S. U. Senarath, J. A. Smith, M. L. Baeck, and J. R. Richardson, 2000: Hydrologic analysis of the Fort Collins, Colorado, flash flood of 1997. J. Hydrol., 228, 82-100.

Ryzhkov, A. V., and D. S. Zrnic, 1998: Polarimetric rainfall estimation in the presence of anomalous propagation. J. Atmos. Oceanic Technol., 15, 1320-1330.

Silverman, B. W., 1986: Density Estimation for Statistics and Data Analysis. Chapman and Hall, 175 pp.

Steiner, M., and J. A. Smith, 2002: Use of three-dimensional reflectivity structure for automated detection and removal of nonprecipitating echoes in radar data. J. Atmos. Oceanic Technol., 19, 673-686.

Straka, J. M., 1996: Hydrometeor fields in a supercell storm as deduced from dual-polarization radar. Preprints, 18th Conf. on Severe Local Storms, San Francisco, CA, Amer. Meteor. Soc., 551-554.

, and D. S. Zrnic, 1993: An algorithm to deduce hydrometeor types and contents from multiparameter radar data. Preprints, 26th Int. Conf. on Radar Meteorology, Norman, OK, Amer. Meteor. Soc., 513-516.

Vivekanandan, J., D. S. Zrnic, S. M. Ellis, R. Oye, A. V. Ryzhkov, and J. Straka, 1999: Cloud microphysics retrieval using Sband dual-polarization radar measurements. Bull. Amer. Me teor. Soc., 80, 381-388.

Wilson, J. W., T. M. Weckwerth, J. Vivekanandan, R. M. Wakimoto, and R. W. Russell, 1994: Boundary layer clear-air radar echoes: Origin of echoes and accuracy of derived winds. J. Atmos. Oceanic Technol., 11, 1184-1206.

Zhang, P., A. V. Ryzhkov, and D. S. Zrnic, 2005: Observations of insects and birds with a polarimetric prototype of the WSR 88D radar. Preprints, $32 d$ Int. Conf. on Radar Meteorology, Albuquerque, NM, Amer. Meteor. Soc., CD-ROM, P6.4

Zrnic, D. S., and A. V. Ryzhkov, 1998: Observations of insects and birds with a polarimetric radar. IEEE Trans. Geosci. Remote Sens., GE-36, 661-668.

— and - 1999: Polarimetry for weather surveillance radar. Bull. Amer. Meteor. Soc., 80, 389-406.

_ and - 2004: Polarimetric properties of chaff. J. Atmos. Oceanic Technol., 21, 1017-1024.

- - J. M. Straka, Y. Liu, and J. Vivekanandan, 2001: Testing a procedure for automatic classification of hydrometeor types. J. Atmos. Oceanic Technol., 18, 892-913.

— , V. M. Melnikov, and A. V. Ryzhkov, 2006: Correlation coefficients between horizontally and vertically polarized returns from ground clutter. J. Atmos. Oceanic Technol., 23, 381-394. 